\title{
PROCESO EXPORTADOR DE EMPRESAS SANTANDEREANAS DE CALZADO A ECUADOR Y VENEZUELA
}

Edgar Javier Gómez Parada. ${ }^{l}$ Gustavo González Sarmiento. ${ }^{2}$

\section{RESUMEN}

Este artículo presenta las características del proceso exportador de las empresas santandereanas de calzado a Ecuador y Venezuela; surge de una investigación realizada por el grupo de investigación en comercio exterior de la Universidad Santo Tomás titulada El Calzado de Santander en los Mercados de Ecuador y Venezuela. Se muestra la participación de los departamentos exportadores de Colombia y se identifican los principales países participantes en los mercados objeto del estudio para reconocer los competidores. Se registra el análisis de variables del proceso exportador, información obtenida a partir de una encuesta aplicada a empresarios exportadores del sector. Se evidencia que Colombia y Santander poseen ventajas en las partidas 6403 y 6402 en los mercados analizados y se detecta que las empresas exportadoras del sector se ubican en primera fase dentro del proceso exportador, caracterizada por modo de exportación indirecta y preferencia por mercados psicológicamente cercanos.

\section{PALABRAS CLAVE}

Calzado santandereano, Proceso exportador, Exportaciones colombianas

\begin{abstract}
This article presents the characteristics of the exporting process of Santander's Footwear companies to Ecuador and Venezuela; it emerges from a study made by the Research Group in International Business of Santo Tomás University entitled El Calzado de Santander en los Mercados de Ecuador y Venezuela. (Santander's Footwear in the markets of Ecuador and Venezuela). It shows the participation of Santander's exporting departments and the main participant countries in the markets object of study to recognize the competitors are identified. The analyses of variables of the exporting process, the data collected from a survey implemented to exporting entrepreneurs of the sector are recorded. It is evidenced that Colombia and Santander have advantages in the portions 6403 and 6402 in the analyzed markets and it is noticed that the exporting companies of the sector are positioned in the first phase within the exporting process, typified by a mode of indirect exportation and preference to psychologically near markets.
\end{abstract}

\section{KEY WORDS}

Santander's Footwear, exporting process, Colombian exports

1 Mg. en Educación. Docente- Investigador Facultad de Negocios Internacionales Universidad Santo Tomás. Grupo de Investigación de la Facultad de Negocios Internacionales.

2 Especialista en Planeación y Administración del Desarrollo Regional. Docente- Investigador Facultad de Negocios Internacionales Universidad Santo Tomás. Grupo de Investigación de la Facultad de Negocios Internacionales. 


\section{Introducción}

La competitividad entendida como la capacidad de una industria de un país o región de ingresar y permanecer en mercados externos, depende directamente de la habilidad para generar valor en un mercado que es cada día más competitivo (Porter, 1991); se hace cada vez más exigente para toda industria, hecho que es bastante evidente para el sector calzado, por ser altamente intensivo en mano de obra, por estar presente en casi todos los países y por las bajas barreras de entrada que se presentan para ingresar a esta actividad productiva.

El sector calzado se caracteriza por (Bekerman, 2000) estar constituido por un conjunto heterogéneo de empresas de distinto tamaño, con predominancia de las PYMES; conjunto variado de productos; competencia por marca, calidad, diseño, eficiencia productiva y es una industria con alta intensidad en mano de obra.

El proceso de ingreso de las empresas a mercados internacionales es lo que se conoce como proceso de internacionalización y se explica desde varias teorías y/o paradigmas; uno de los más aceptados, y que se asume como marco para el presente trabajo investigativo, es el modelo gradualista o incremental, más conocido como modelo de Uppsala ${ }^{3}$. Este modelo propone que las empresas deciden internacionalizarse a partir de de pasos incrementales, que conlleva una serie de decisiones donde los principales determinantes son el conocimiento del mercado y los recursos; y presenta cuatro fases: exportaciones esporádicas e irregulares, exportaciones vía agente independiente, filiales comerciales y filiales de producción.

De acuerdo a Olivares y Suárez, (2006) y Aranda y Montoya (2006), que ofrecen una explicación del modelo; en una fase previa (a las expuestas anteriormente) la empresa se consolida en mercados internos sin realizar exportaciones. En la primera fase, la empresa realiza exportaciones a países del extranjero psicológicamente cercanos y de un modo indirecto (a través de comercializadoras internacionales $u$ otros mecanismos), lo cual requiere bajo compromiso en el proceso exportador; las exportaciones realizadas son bajas (en volumen de ventas) y esporádicas (estáticas). El aprendizaje sobre el mercado, sobre la gestión y sobre negocios internacionales es bajo o nulo. En una fase posterior las exportaciones las realiza la empresa de manera directa y a distancias medias (geográfica y psicológica), requiere de la empresa mayor compromiso y adquiere experiencia y aprendizaje del mercado externo, de los negocios; de marketing y de gestión exportadora. En etapas más avanzadas, se exporta de manera directa; a múltiples mercados y a grandes distancias. Dentro de

3 A partir de trabajos realizados principalmente por Johanson y Vahlne desde el Department of Business Administration, Uppsala a partir de 1975 y publicados en diversos artículos y working papers. 
estas dos últimas etapas aparecen varias formas de internacionalización como son las franquicias, filiales comerciales, empresas mixtas; hasta llegar al establecimiento de unidades productivas en mercados externos que caracteriza la última fase.

Aparece como variable que complementaría a la explicación del modelo gradualista la distancia psicológica del mercado extranjero al cual se exporta. La distancia psicológica hace referencia a la similitud en aspectos culturales y sociales, que hace que las relaciones, incluidas las comerciales, se den de una manera fluida, entre dos naciones (Czinkota, 2007). La distancia psicológica comprende los factores que facilitan los flujos de información entre la empresa y el mercado y, por tanto, conlleva a una comprensión del mercado. Entre ellos están las diferencias en el lenguaje, la cultura, los sistemas políticos, el nivel de capital humano, el nivel de desarrollo industrial y las prácticas empresariales. Normalmente, está correlacionada con la distancia geográfica, aunque la distancia psicológica no es constante en el tiempo. En ocasiones existen diferencias que hace que naciones cercanas geográficamente sean lejanas psicológicamente; entre ellos pueden estar aspectos políticos y de política comercial. En las primeras etapas del proceso de internacionalización las exportaciones se realizan a mercados psicológica y geográficamente cercanos.

Para Colombia los mercados de Ecuador y Venezuela, además de ser geográficamente cercanos por estar ubicados en las fronteras, son también cercanos psicológicamente por múltiples similitudes culturales, del lenguaje, sistemas políticos e incluso en los sistemas de producción. Tradicionalmente se han considerado como mercados naturales para Colombia.

Dentro del proceso exportador ${ }^{4}$ se incluyen para este estudio, variables relacionadas con el marketing internacional (Soret, 2004) como forma de contacto con los clientes, mecanismos para dar a conocer el producto y forma de pago, y actividades logísticas (López, 2004) como medio de transporte y ubicación del producto. La recolección de información se realizó a partir de la aplicación de una encuesta a empresarios exportadores de calzado a los países de Ecuador y Venezuela.

Según las revisiones realizadas no se encuentran estudios que proporcionen información sobre el proceso exportador y posicionamiento del sector calzado de Santander respecto a mercados externos; menos aún en mercados como Ecuador y Venezuela.

$4 \quad$ El proceso exportador es una de las formas de internacionalización. El proceso exportador se interpreta como un conjunto de actividades logísticas y de marketing incluidas dentro de la distribución física internacional. 


\section{Metodología}

Es un estudio de tipo descriptivo, que se basa en fuentes de información primaria y secundaria. Las fuentes primarias son las empresas (unidad de análisis de información) que suministran información a partir del diligenciamiento de la encuesta, enfocada al proceso exportador hacia los mercados de Ecuador y Venezuela. Dentro del proceso exportador se tuvo en cuenta variables de marketing y logística internacional; como mecanismo de exportación, permanencia exportadora en el mercado objetivo, tamaño de las exportaciones, línea de producto, tipo de transporte, ubicación del producto, contacto con los clientes, formas de pago. Se aplicó la encuesta a 50 empresas exportadoras de calzado a dichos mercados. La técnica utilizada para el análisis de información primaria fue la estadística descriptiva.

El estudio investigativo abarcó las fases de elaboración de marco referencial, detección y obtención de datos de estadísticos, principalmente el Sistema Estadístico de Comercio Exterior DIAN - SIEX de Colombia; Banco de Comercio Exterior de Venezuela (BANCOEX) y el Instituto Nacional de Estadística y Censos de Ecuador (INEC). Se estableció correlación entre los datos estadísticos e interpretación de los mismos. La tercera fase consistió en la recolección de la información primaria.

\section{Resultados}

Las mercancías que se integran al flujo del comercio internacional se clasifican con base en el sistema armonizado de designación y codificación de mercancías, el cual ha sido adoptado por la mayoría de los países, se denomina arancel armonizado. Los productos de calzado se ubican dentro del capítulo 64 y está constituido por 6 partidas arancelarias (posiciones a 4 dígitos) que se describen en la Tabla 1.

Tabla 1. Descripción de partidas arancelarias del sector calzado

\begin{tabular}{cl}
\hline PARTIDA & \multicolumn{1}{c}{ DESCRIPCIÓN } \\
\hline 6401 & $\begin{array}{l}\text { Calzado impermeable con suela y parte superior de caucho o plástico, cuya parte } \\
\text { superior no se haya unido a la suela por costura o por medio de remaches, clavos, } \\
\text { tornillos, espigas o dispositivos similares, ni se haya formado con diferentes } \\
\text { partes unidas de la misma manera. }\end{array}$ \\
\hline 6402 & Los demás calzados con suela y parte superior de caucho o plástico \\
\hline 6403 & $\begin{array}{l}\text { Calzado con suela de caucho, plástico, cuero natural o regenerado y parte superior } \\
\text { de cuero natural. }\end{array}$ \\
\hline 6404 & $\begin{array}{l}\text { Calzado con suela de caucho, plástico, cuero natural o regenerado y parte superior } \\
\text { de materia textil. }\end{array}$ \\
\hline 6405 & $\begin{array}{l}\text { Los demás calzados. } \\
6406\end{array}$ \\
$\begin{array}{l}\text { Partes de calzado (incluidas las partes superiores fijadas a las palmillas distintas } \\
\text { de la suela); plantillas, taloneras y artículos similares, amovibles; polainas y } \\
\text { artículos similares, y sus partes }\end{array}$ \\
\hline
\end{tabular}

Fuente: Elaboración propia a partir de Arancel Armonizado Colombiano 


\subsection{Situación del sector calzado de Santander}

El sector calzado en Santander es considerado estratégico por la cantidad de empresas existentes, algunas registradas formalmente ante la Cámara de Comercio, otras funcionan de manera informal como talleres, famiempresas o empresas satélites; que han sido tradicionalmente generadoras de empleo en la región.

Tabla 2. Empresas registradas en la Cámara de Comercio de Bucaramanga año 2006

\begin{tabular}{lcccc}
\hline \multicolumn{1}{c}{ Actividad económica } & $\begin{array}{c}\text { No. } \\
\text { Empresas }\end{array}$ & Empleos & $\begin{array}{c}\text { Activos } \\
\text { (en pesos) }\end{array}$ & $\begin{array}{c}\text { Ventas Netas } \\
\text { (en pesos) }\end{array}$ \\
\hline Fabricación de calzado & 63 & 210 & 339.447 .580 & 498.210 .500 \\
\hline $\begin{array}{l}\text { Fabricación de calzado de } \\
\text { caucho, excepto deportivo }\end{array}$ & 10 & 20 & 15.560 .000 & 23.000 .000 \\
\hline $\begin{array}{l}\text { Fabricación de calzado } \\
\text { deportivo }\end{array}$ & 20 & 70 & 855.401 .350 & 901.223 .400 \\
\hline $\begin{array}{l}\text { Fabricación de partes de } \\
\text { calzado }\end{array}$ & 26 & 86 & 721.252 .850 & 918.776 .000 \\
\hline Fabricación de calzado NCP & 7 & 20 & 42.186 .020 & 86.394 .175 \\
\hline
\end{tabular}

Fuente: Elaboración propia a partir de datos suministrados por Cámara de Comercio de Bucaramanga.

Las exportaciones nacionales de calzado presentan aumentos significativos entre los años 2003 - 2006 (245\%), de manera sostenida en los cuatro años. Santander también creció en las exportaciones del sector calzado en este período, sin embargo, el ritmo no es el mismo que el presentado a nivel nacional. El crecimiento departamental en los cuatro años analizados es del 50\% frente al 245\% del nacional; por tanto Santander viene perdiendo paulatinamente participación en las exportaciones nacionales.

Tabla 3. Exportaciones de calzado de Santander. Miles de US FOB

\begin{tabular}{lcccc}
\hline CALZADO & $\mathbf{2 0 0 3}$ & $\mathbf{2 0 0 4}$ & $\mathbf{2 0 0 5}$ & $\mathbf{2 0 0 6}$ \\
\hline Exportaciones nacionales & $28.932,0$ & $43.067,8$ & $61.927,9$ & $70.961,3$ \\
\hline Exportaciones Santander & $5.037,3$ & $6.744,0$ & $6.969,8$ & $7.411,3$ \\
\hline $\begin{array}{l}\text { \% Participación de } \\
\text { Santander }\end{array}$ & 17,4 & 15,7 & 11,3 & 10 '4 \\
\hline
\end{tabular}

Elaboración propia a partir de: DIAN - Siex. 
Gráfico 1. Comportamiento exportaciones de calzado. Colombia y Santander Elaboración propia

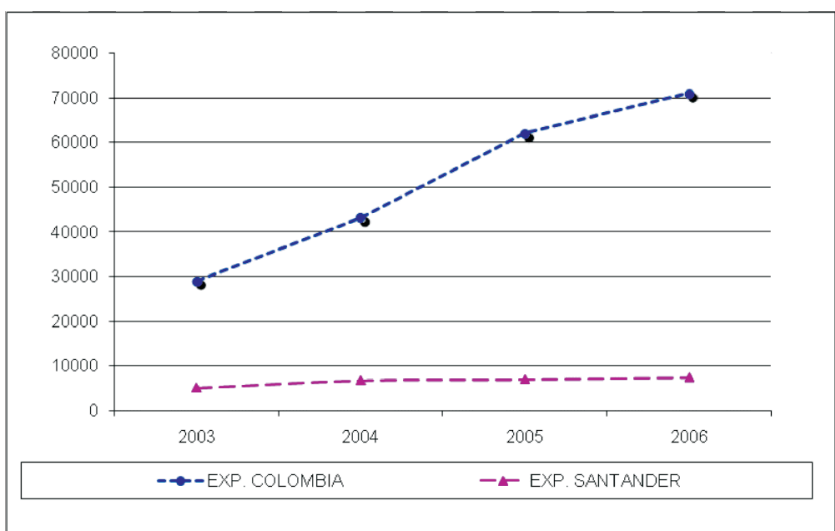

Ecuador es el principal país destino de las exportaciones de calzado de Santander; en el período 2003 - 2006, liderazgo que mantuvo durante todo el tiempo. El comportamiento de las exportaciones a Ecuador presentó un incremento del 47,5\% entre el 2003 y el 2004 y de 20,96\% entre el 2004 y el 2005. Del 2005 al 2006 sufrió una disminución del 16,26\%.

Tabla 4. Destino de las exportaciones de calzado de Santander. US FOB ${ }^{5}$

\begin{tabular}{lrrrr}
\hline PAÍS & \multicolumn{1}{c}{2003} & \multicolumn{1}{c}{2004} & \multicolumn{1}{c}{2005} & \multicolumn{1}{c}{2006} \\
\hline Ecuador & $1,241,065$ & $1,782,769$ & $2,156,376$ & $1,805,749$ \\
\hline Puerto Rico & 1,240638 & $1,071,919$ & $1,110,845$ & $1,252,786$ \\
\hline Panamá & 708,078 & 989,202 & 741,422 & 731,698 \\
\hline Venezuela & 406,760 & 926,312 & 833,831 & $1,614,521$ \\
\hline Estados Unidos & 556,492 & 694,245 & 714,097 & 376,627 \\
\hline Antillas holandesas & 174,160 & 161,138 & 244,683 & 222,981 \\
\hline Araba & 144,893 & 279,553 & 233,971 & 122987 \\
\hline Martinico & 65,586 & 194,633 & 173,416 & 196679 \\
\hline Reino Unido & 61,665 & 90,807 & 89,309 & 119358 \\
\hline Barbados & 89,131 & 69,772 & 88,410 & 53690 \\
\hline Bahamas & 52,929 & 9,130 & 61,627 & 60053 \\
\hline Guadalupe & 79,446 & 25,484 & 57,299 & 72046 \\
\hline
\end{tabular}

5 Precio de las mercancías puestas a bordo en el puerto de embarque (FOB: F REE ON BOARD, libre a bordo). 


\begin{tabular}{lrrrr}
\hline PAÍS & \multicolumn{1}{c}{2003} & \multicolumn{1}{c}{2004} & \multicolumn{1}{c}{2005} & \multicolumn{1}{c}{2006} \\
\hline Guatemala & 65,185 & 87,236 & 57,241 & 16530 \\
\hline Costa Rica & 34,451 & 111,105 & 53,018 & 96500 \\
\hline Perú & 4,200 & 48,582 & 44,670 & 92028 \\
\hline República Dominicana & 2,087 & 2,624 & 37,057 & 31049 \\
\hline Santa Lucía & 22,059 & 8,862 & 32,404 & 17203 \\
\hline Trinidad y Tobago & 3,528 & 25,422 & 20,057 & 41050 \\
\hline Zona Franca Cúcuta & & 44,041 & 42,849 & 199,946 \\
\hline Total & 5,037300 & 6,744000 & 6,969800 & 7,411300 \\
\hline
\end{tabular}

Fuente: Elaboración a partir: DIAN - Siex

Gráfico 2. Comportamiento de las exportaciones de calzado de Santander a Ecuador

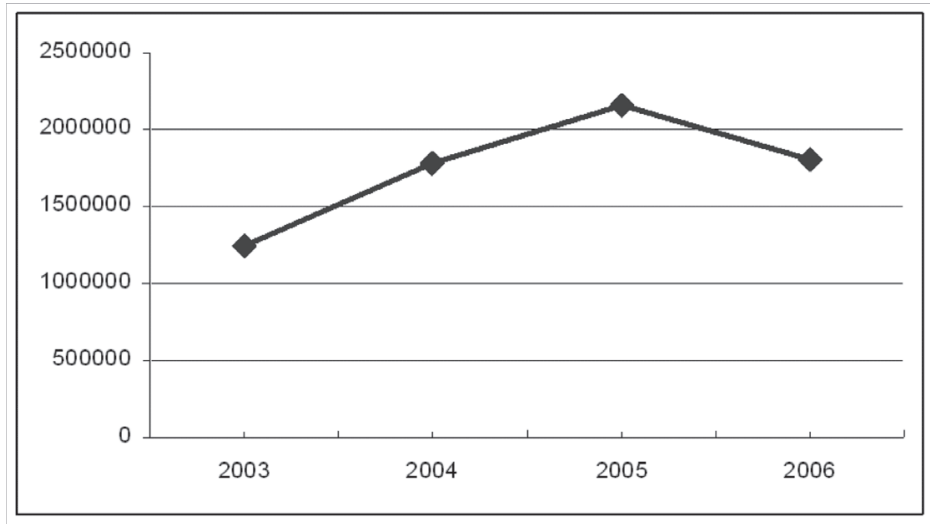

Fuente: Elaboración propia

Venezuela en el año 2006 fue el segundo país destino de las exportaciones de calzado de Santander, mostrando un aumento significativo respecto al 2005, de 100\%. Del 2003 al 2006 el aumento fue de 400\%. Esto en alguna medida se explica por la caída de la producción de calzado en Venezuela (Coindustria, 2005). 
Gráfico 3. Exportaciones de calzado de Santander a Venezuela

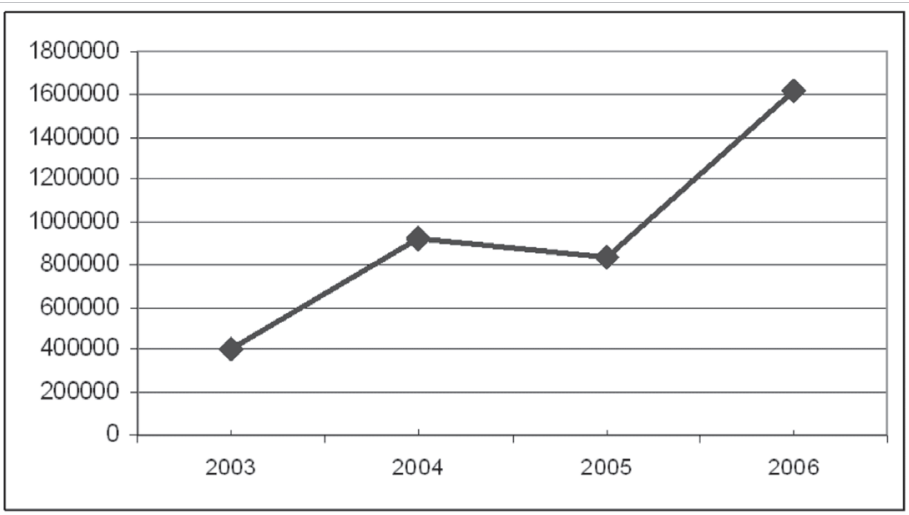

Fuente: Elaboración propia

La partida arancelaria con mayor participación en las exportaciones de calzado de Santander fue la 6403 (calzado de cuero en la parte superior) con el 67,7\% del valor de las exportaciones. El segundo renglón correspondió a la partida 6402 (demás calzados de caucho o plástico) con el $22,3 \%$. Las demás partidas tuvieron una baja participación en las exportaciones. Es decir, que la 6403 y la 6402 son las partidas con mayor potencial exportador.

Tabla 5. Exportaciones de calzado de Santander por partidas. Año 2006. US FOB

\begin{tabular}{ccc}
\hline PARTIDA & Valor & \% Part. \\
\hline 6402 & $1^{\prime} 650.000$ & 22,3 \\
\hline 6403 & $5 ’ 017.700$ & 67,7 \\
\hline 6404 & 199.100 & 2,7 \\
\hline 6405 & 225.800 & 3 \\
\hline 6406 & 318.700 & 4,3 \\
\hline TOTAL & 7'411.300 & 100 \\
\hline
\end{tabular}

Fuente: Elaboración a partir: DIAN - Siex 
Dentro de las exportaciones colombianas de calzado, la partida 6403 participa con el $52 \%$ de todo el sector (Tabla 5). La partida 6402 contribuye con el 23\%; Colombia también exporta una cantidad significativa de partes de calzado $(18 \%)$.

Tabla 6. Exportaciones de calzado de Colombia por partidas. Año 2006. US FOB

\begin{tabular}{crc}
\hline PARTIDA & \multicolumn{1}{c}{ Valor } & \% Part. \\
\hline 6401 & $8^{\prime} 75.270$ & $1 \%$ \\
\hline 6402 & $16^{\prime} 129.853$ & $23 \%$ \\
\hline 6403 & $36^{\prime} 832.537$ & $52 \%$ \\
\hline 6404 & $3^{\prime} 267.388$ & $5 \%$ \\
\hline 6405 & 856.254 & $1 \%$ \\
\hline 6406 & $13^{\prime} 000.017$ & $18 \%$ \\
\hline Total & $70^{\prime} 961.319$ & \\
\hline
\end{tabular}

Fuente: Elaboración a partir: DIAN - Siex

\subsection{Posicionamiento de Santander en los mercados de Ecuador y Venezuela}

Valle del Cauca es el Departamento con mayor participación en las exportaciones de calzado a Ecuador con el 27\%; le sigue Antioquia con $21 \%$ y Santander con $17 \%$. Valle del Cauca presenta una ventaja comparativa respecto a las demás regiones, por encontrarse geográficamente más cerca de Ecuador, factor que incide en la disminución del costo de transporte que fácilmente se traduce en ventajas competitivas frente a otras regiones de Colombia.

En las exportaciones a Venezuela el Departamento con mayor participación es Norte de Santander, que registra el 43\% del total exportado; esta situación, se explica en parte por la cercanía de esta zona con Venezuela. Se destacan además Valle del Cauca (23\%), Antioquia (16\%) y Cundinamarca (6\%). Santander solo exporta el 5\% a Venezuela, a pesar de encontrarse más cerca geográficamente que otras regiones. 
Tabla 7. Departamentos exportadores de calzado a Ecuador y Venezuela.

Año 2006. US FOB

\begin{tabular}{lcccr}
\hline \multirow{2}{*}{ REGIÓN } & \multicolumn{2}{c}{ ECUADOR } & \multicolumn{2}{c}{ VENEZUELA } \\
\cline { 2 - 5 } & \multicolumn{1}{c}{ Valor } & $\%$ Part. & \multicolumn{1}{c}{ Valor } & $\%$ Part. \\
\hline Norte de Santander & 74.203 & $1 \%$ & 13.464 .992 & $43 \%$ \\
\hline Valle del Cauca & 2.985 .684 & $27 \%$ & 7.175 .901 & $23 \%$ \\
\hline Antioquia & 2.285 .037 & $21 \%$ & 4.924 .062 & $16 \%$ \\
\hline Cundinamarca & 1.280 .843 & $12 \%$ & 1.803 .451 & $6 \%$ \\
\hline Santander & 1.805 .749 & $17 \%$ & 1.614 .521 & $5 \%$ \\
\hline Bogotá & 1.744 .297 & $16 \%$ & 1.089 .438 & $3 \%$ \\
\hline Otros & 598.622 & $6 \%$ & 1.278 .368 & $4 \%$ \\
\hline Total & 10.774 .435 & & 31.350 .733 & \\
\hline
\end{tabular}

Fuente: Elaboración a partir: DIAN - Siex

Se observa que Santander exporta más a Ecuador que a Venezuela, a pesar de la cercanía geográfica; igual sucede con Valle del Cauca que exporta tres veces más a Venezuela que a Ecuador. Las exportaciones colombianas de calzado a Venezuela son mayores que a Ecuador en una relación de 1 a 3.

La mayor participación en las exportaciones del sector calzado tanto para Colombia, como para Santander, corresponden a las partidas 6403 y 6402 respectivamente. El análisis en este artículo se continúa con estas partidas.

Venezuela realizó mayores importaciones de calzado que Ecuador (relación de 3 a 1), en el 2006. Es decir, que el tamaño del mercado venezolano es más atractivo que el ecuatoriano.

Tabla 8. Importaciones de calzado realizadas por Ecuador y Venezuela en el sector calzado. Año 2006. US FOB

\begin{tabular}{cccrc}
\hline \multirow{2}{*}{ PARTIDA } & \multicolumn{2}{c}{ VENEZUELA } & \multicolumn{2}{c}{ ECUADOR } \\
\cline { 2 - 5 } & \multicolumn{1}{c}{ Valor } & \% Part. & \multicolumn{1}{c}{ Valor } & $\%$ Part. \\
\hline 6401 & 6278796 & $2 \%$ & 1093000 & $1 \%$ \\
\hline 6402 & 54695505 & $19 \%$ & 55881000 & $56 \%$ \\
\hline 6403 & 53917536 & $17 \%$ & 26023000 & $26 \%$ \\
\hline 6404 & 97213756 & $32 \%$ & 11973 & $13 \%$ \\
\hline 6405 & 76523857 & $25 \%$ & 1312 & $1 \%$ \\
\hline 6406 & 14162777 & $5 \%$ & 3080000 & $3 \%$ \\
\hline Total & $\mathbf{3 0 2 7 5 6 2 2 7}$ & & $\mathbf{9 9 3 6 1 0 0 0}$ & \\
\hline
\end{tabular}

Fuente: Elaboración a partir de: Venezuela: Banco de Comercio Exterior de Venezuela: Bancoex. Ecuador: Banco Central de Ecuador. 
Las importaciones de Ecuador en la partida 6402 provienen de China (42,9\%); Panamá $(20,7 \%)$ y Brasil (13,8\%) principalmente. Colombia participó solamente con el 3,4\%. En la partida 6403 las importaciones de dicho país están lideradas por Colombia con (23\%) seguido por Panamá (20\%) y Brasil (15,6\%); además tienen presencia en el mercado Perú, Estados Unidos y China.

Tabla 9. Origen de las importaciones ecuatorianas de la partida 6402 y 6403 . Año 2006 US FOB

\begin{tabular}{lrrrr}
\hline PAÍ́S & \multicolumn{2}{c}{$\mathbf{6 4 0 2}$} & \multicolumn{2}{c}{$\mathbf{6 4 0 3}$} \\
\hline Valor & \% Part. & Valor & \% Part. \\
\hline China & 23968 & 42,9 & 1642 & 6,31 \\
\hline Panamá & 11553 & 20,7 & 5237 & 20,12 \\
\hline Brasil & 7698 & 13,8 & 4056 & 15,59 \\
\hline Zona Franca de Ecuador & 6910 & 12,4 & 4220 & 16,22 \\
\hline Estados Unidos & 3203 & 5,7 & 1890 & 7,26 \\
\hline Colombia & 1921 & 3,4 & 6006 & 23,08 \\
\hline Otros & 628 & 1,1 & 2971 & 11,42 \\
\hline Total & 55881 & 100 & 26023 & 100 \\
\hline
\end{tabular}

Fuente: Elaboración a partir de: Banco central de Ecuador.

En la partida 6403 la mayor participación corresponde a Colombia $(23,08 \%)$; como competidores tiene a Panamá, Brasil y Zona franca de Ecuador. Por consiguiente, el posicionamiento de Colombia en el mercado Ecuatoriano es altamente favorable en esta partida. En el subsector 6402 la situación es menos positiva para Colombia dado que sólo aporta el 3,4\% de las importaciones ecuatorianas, con amplio distanciamiento respecto a China país líder en esta línea.

Santander exportó productos de la partida 6403 por valor de 1'084.726 dólares en el año 2006 a Ecuador, para una participación del 18\% del total aportado por Colombia a dicho mercado. En la partida 6402 Santander exportó a Ecuador 662.740 dólares para una participación del $34 \%$ del total exportado por Colombia al mercado ecuatoriano.

Respecto al comportamiento de las importaciones venezolanas, el valor de las compras provenientes de Colombia es muy similar en las partidas 6402 y 6403 . En la 6402, Colombia ocupa el primer lugar y aporta el 21,7\%, participan además Panamá $(16,4 \%)$, Brasil (16,3\%), Indonesia (15,8\%), y China $(17,0 \%)$, entre otros. En el renglón 6403 la mayor contribución es de Brasil (22,1\%), seguido por China (20,1\%) y Colombia $(19,9 \%)$ en el tercer lugar. 
Tabla 10. Origen de las importaciones venezolanas de la partida 6402 y 6403 . Año 2006. US FOB

\begin{tabular}{lrrrr}
\hline \multicolumn{1}{c}{ PAÍ́S } & \multicolumn{2}{c}{$\mathbf{6 4 0 2}$} & \multicolumn{2}{c}{$\mathbf{6 4 0 3}$} \\
\hline Valor & \% Part. & \multicolumn{1}{c}{ Valor } & \% Part. \\
\hline Colombia & 11869 & 21,7 & 10711 & 19,9 \\
\hline Panamá & 8941 & 16,4 & 1833 & 3,4 \\
\hline Brasil & 8905 & 16,3 & 11890 & 22,1 \\
\hline Indonesia & 8613 & 15,8 & 4234 & 7,9 \\
\hline China + Hong Kong & 7756 & 17,0 & 10816 & 20,1 \\
\hline Malasia & 4078 & 7,5 & 0 & \\
\hline Otros & 4499 & 5,4 & 5705 & 10,4 \\
\hline España & 0 & & 5270 & 9,8 \\
\hline Vietnam & 0 & & 3459 & 6,4 \\
\hline TOTAL & 54660 & 100,0 & 53918 & 100 \\
\hline
\end{tabular}

Fuente: Elaboración a partir de: Banco de Comercio Exterior de Venezuela. Bancoex.

El posicionamiento de Colombia en las partidas 6403 y 6402 en el mercado venezolano es ampliamente favorable con participaciones de 19,9\% y 21,7\% respectivamente. Se observa una fuerte competencia de parte de Brasil y China + Hong Kong, con porcentajes muy cercanos en participación; esto aplica para ambas partidas.

Las exportaciones de Santander en la partida 6403 a Venezuela alcanzaron un valor de 743.743 dólares Fob, que equivale al 7,5\% de lo exportado por Colombia a dicho mercado; es decir que se presenta desventaja frente a otras regiones, como Norte de Santander, Valle del Cauca y Antioquia, en este renglón. En la partida 6402 se exportó un valor de 511.370 dólares Fob, para una participación de 4,3\% de las exportaciones colombianas a ese país.

Aunque Colombia es fuerte en la participación de las partidas 6403 y 6402 en el mercado venezolano, el aporte de Santander frente a otras regiones de Colombia es bajo; por consiguiente no aprovecha la cercanía geográfica o distancia psicológica respecto a Venezuela.

El desempeño de las exportaciones de calzado de Santander, en las partidas 6402 y 6403 es más favorable en el mercado ecuatoriano respecto al venezolano, esta situación es paradójica por cuanto Venezuela está más cerca geográficamente a Santander. Es de señalar además que el tamaño del mercado en la partida 6403 es el doble en Venezuela respecto a Ecuador, hecho que refleja desaprovechamiento de oportunidades y desconocimiento del comportamiento del mercado por parte de los empresarios del sector calzado. 


\subsection{Perfil del proceso exportador a Ecuador y Venezuela}

No se pretende explicar el proceso exportador de las empresas del calzado en su totalidad, sin embargo, es un acercamiento al conocimiento de la gestión exportadora de los empresarios de la región. En la encuesta se incluyeron algunos ítems relacionados con variables de marketing internacional y de logística internacional.

En las líneas siguientes se presentan los resultados de la encuesta aplicada a 50 empresarios exportadores de calzado de Santander; a los mercados de Ecuador y/o Venezuela. Las empresas se seleccionaron según información suministrada por $\mathrm{ACICAM}^{6}$ seccional Santander.

El $90 \%$ de las empresas envía calzado a Venezuela y el 62\% a Ecuador. El 50\% de las empresas exportan tanto a Ecuador como a Venezuela; el $40 \%$ solo a Venezuela, el $12 \%$ solo a Ecuador.

\section{Mecanismo de exportación}

Para las exportaciones, las empresas prefieren utilizar los servicios de una comercializadora internacional (62\%), en algunos casos las efectúa directamente $(22 \%)$; en otros se combinan ambos mecanismos (16\%). Es decir, que la tendencia dominante es realizar las exportaciones de una manera indirecta. Se puede apreciar que las empresas en su expansión internacional a Ecuador y Venezuela usan modalidad de exportación indirecta, que es un modo de entrada que implica bajo compromiso de la empresa, derivado de la baja experiencia en el mercado (Olivares, 2007).

\section{Permanencia exportadora}

El $77,80 \%$ de las empresas cuenta con una experiencia entre 1 y 3 años de exportar productos de calzado a Venezuela y el 4,44\% lleva menos de un año en procesos de negociación con el país fronterizo. Otro 4,44\% tienen amplia trayectoria en exportación de calzado a ese país con más de 5 años de experiencia. Esto refleja que la permanencia exportadora de los empresarios de Santander en Venezuela es muy baja hecho que afecta directamente la competitividad, debido a que no se logra entrar en fase avanzada (Olivares, 2006), donde se observe el aprovechamiento de distancia psicológica cercana.

6 Asociación colombiana de industriales del calzado, el cuero y sus manufacturas. Principal gremio que agrupa a los empresarios del calzado en Santander. 
Tabla 11. Permanencia exportadora empresas de Santander a Ecuador y Venezuela

\begin{tabular}{lcc}
\hline \multicolumn{1}{c}{ Años } & $\begin{array}{c}\text { Permanencia } \\
\text { exportadora a } \\
\text { Ecuador }\end{array}$ & $\begin{array}{c}\text { Permanencia } \\
\text { exportadora a } \\
\text { Venezuela }\end{array}$ \\
\hline Menos de 1 año & $6,45 \%$ & $4,44 \%$ \\
\hline Entre 1 - 3 años & $61,29 \%$ & $77,80 \%$ \\
\hline Más de 3 y 5 años & $19,36 \%$ & $13,32 \%$ \\
\hline Más de 5 años & $12,90 \%$ & $4,44 \%$ \\
\hline Total & $100 \%$ & $100 \%$ \\
\hline
\end{tabular}

Fuente: autores a partir de encuestas.

El $61,29 \%$ de las empresas llevan entre 1 y 3 años de exportar a Ecuador y el $6,45 \%$ tiene menos de un año de experiencia en dicho mercado. El 19,36\% de las empresas tiene una trayectoria entre 3 y 5 años y sólo el $12,90 \%$ lleva más de 5 años de posicionar sus productos en el mercado ecuatoriano. Tal como sucede con Venezuela, en el caso de Ecuador, la permanencia exportadora también es baja y no se aprovecha la distancia psicológica.

\section{Tamaño de las exportaciones}

El volumen de exportación a Venezuela en pares de calzado es bajo; el promedio anual es de 6444 pares exportados por empresa. Anual, el 53,49\% de las empresas exportan menos de 3000 pares al año y el 9,30\% exportan más de 10000 pares.

A Ecuador se exporta un volumen más reducido; el promedio de pares es de 3607 anuales por empresa. El 44,45\% de las empresas exportan 1000 pares o menos. El $14,8 \%$ de fábricas exportan más de 5000 pares al mercado ecuatoriano.

El promedio de exportación por empresa es poco significativo, hecho relacionado directamente con el tamaño de las empresas; baja permanencia exportadora por empresa en cada mercado, escasa experiencia en manejo y conocimiento del mercado e información.

Al contrastar las cifras estadísticas mostradas en la primera sección, con la información proporcionada por las empresas, se encuentra una aparente contradicción. El valor de exportaciones a Ecuador es mayor que a Venezuela, sin embargo, en número de pares de calzado es más alto a Venezuela que al mercado ecuatoriano en una proporción aproximada de 2 a 1. Esto puede explicarse a partir de la diferencia de precios a cada mercado; se supone que los precios de venta de calzado a Ecuador son mayores frente al calzado que se vende a Venezuela. 


\section{Línea de producto}

La línea que más se exporta es calzado para dama $(60 \%)$ seguido de zapato infantil tanto para niño como para niña (28\%) y calzado para caballero (26\%); y el estilo de calzado predominante es zapatilla y sandalia.

\section{Tipo de transporte}

El transporte más utilizado para enviar productos a Ecuador y Venezuela es el terrestre, empleado por el $96 \%$ de las empresas. También se emplea transporte aéreo y en algunos casos esporádicos transporte marítimo.

\section{Ubicación del producto}

El $67,75 \%$ de empresas que exportan a Ecuador ubican el calzado en Ipiales; que es una ciudad fronteriza localizada en Colombia. En este caso, el producto se lleva a ese lugar para ser internacionalizado en el mercado ecuatoriano por parte del importador, o de la comercializadora internacional.

La mayoría de empresas (86,70\%) que realizan exportaciones a Venezuela ubican el calzado en Cúcuta, que es una ciudad fronteriza localizada en Colombia; y de allí el importador o la comercializadora internacional, procede a la internalización del producto al mercado venezolano.

\section{Contacto con los clientes}

La forma predominante de establecer contactos con los clientes es a través de las ferias que se ha constituido en una buena estrategia para establecer relaciones comerciales internacionales; algunas de ellas organizadas por los gremios como ACICAM y ASOINDUCALS 7 . Se destacan otras estrategias para conseguir clientes internacionales como visita a puntos de fábrica, misiones comerciales, referencias de otros empresarios y cámaras de integración.

El producto se da a conocer mediante muestra física (88\%) en ferias y directamente en la fábrica. También se emplean los medios electrónicos como página Web, catálogos virtuales y correos electrónicos. Otra estrategia es el manejo de catálogos físicos.

$7 \quad$ Asociación de industriales del calzado y similares 


\section{Formas de pago}

Se utiliza principalmente dos formas de pago: hasta 30 días y de contado. Algunos exportadores pactan plazos hasta 90 días. Al 68\% de las empresas exportadoras les pagan con moneda colombiana (peso colombiano). Aunque para los negocios internacionales la moneda más utilizada es el dólar. En el caso de los exportadores de calzado del Santander tan sólo el 34\% negocia bajo esta moneda. En otros casos menos significativos se pacta en moneda venezolana o ecuatoriana.

El nivel de confiabilidad que los empresarios exportadores de calzado tiene en sus clientes extranjeros venezolanos y ecuatorianos es bueno. El 74\% deposita en sus compradores una alta confiabilidad y el 18\% lo expresa como media; en este último caso la confiabilidad ha disminuido por atrasos en los pagos principalmente.

Diversos factores tienen en cuenta los empresarios para decidir la realización del proceso de exportación. Para el $90 \%$ el factor primordial es la forma de pago; siguen en orden de importancia, las referencias del cliente y la estabilidad en el negocio.

La mayoría de las empresas encuentran más experiencias positivas que negativas en los procesos de exportación de calzado que han realizado a Ecuador y Venezuela. Según opinión de los empresarios los principales aspectos positivos se basan en el aumento de ingresos $(22 \%)$ y posicionamiento de la marca $(18 \%)$.

También se encuentran algunas experiencias negativas; como la inestabilidad cambiaria; las barreras arancelarias, problemas aduaneros, devolución de la mercancía, falsificación de documentos e incumplimientos por parte de los compradores extranjeros.

Son variadas las estrategias que utilizan los exportadores de calzado de Santander para conocer las tendencias de la moda y de allí generar los diseños del calzado que ofertan al mercado internacional. La que más destacan los empresarios es el uso de Internet y revistas especializada, asistencia a ferias y viajes al exterior.

Estas evidencias dejan ver que el camino por recorrer por parte de los empresarios es largo, que las empresas están en proceso de aprendizaje y maduración en los procesos de exportación y que la vocación exportadora del sector calzado de Santander es bastante baja. Son muchos los esfuerzos por hacer para consolidar un verdadero sector exportador, como también para profundizar sobre aspectos particulares y específicos de los negocios internacionales.

\section{Conclusiones}

Las mayores exportaciones del sector calzado de Santander se realizan a mercados geográfica y psicológicamente cercanos, por las similitudes culturales, de lenguaje 
y de especialización productiva, entre otros, como son Ecuador y Venezuela. Sin embargo, la internacionalización de las empresas del sector en dichos mercados, está en fase de consolidación, ya que se aprecia una alta tendencia al modo de exportación indirecta y la permanencia exportadora es baja.

La forma de exportación predominante es a través de comercializadoras internacionales, es decir, de manera indirecta. Esto evita el alto compromiso de la empresa en los negocios internacionales e impide el aprendizaje y la experiencia el proceso de internacionalización además que dificulta la captación de mecanismos claves como vía de acceso a los mercados internacionales.

Estas realidades ubican al sector calzado en la primera fase del proceso de internacionalización, de acuerdo al modelo Upssala; caracterizada principalmente por mayor concentración de exportaciones a mercados psicológicamente cercanos y por modo de exportación indirecta. Esto evidencia el bajo compromiso de los empresarios exportadores del sector en el proceso de exportación y poco control en las operaciones de internacionalización del producto.

El promedio de exportación a los mercados de Ecuador y Venezuela es bastante bajo (6400 pares por empresa anualmente); al igual que la permanencia exportadora de las empresas (el 77\% lleva menos de tres años exportando a estos mercados); esto unido al modo de exportación indirecta, reforzado con el hecho de que el producto se ubica principalmente en ciudades fronterizas colombianas (Cúcuta e Ipiales); evidencian que no se logra conocimiento del mercado ni aprendizaje en el proceso de exportación por parte de las empresas; variables fundamentales para poder avanzar hacia fases posteriores en el proceso de internacionalización.

El logro de mayor participación en los mercados actuales y acceso a mercados más exigentes que ofrezcan mejores oportunidades, se logra en la medida que se supere con éxito la primera fase del proceso de internacionalización. Ello exige a las empresas mayor control y compromiso en las operaciones de exportación, de acuerdo al modelo de Upssala, el conocimiento y la experiencia son los determinantes para consolidarse en los mercados actuales y expandirse a otros mercados.

La participación de Santander en las exportaciones de calzado es muy baja en relación a otras regiones del país y no se aprovechan ventajas comparativas respecto a Venezuela en partidas donde Colombia presenta fortalezas y alta participación respecto a otros países del hemisferio.

La relación de tamaño del mercado entre Venezuela y Ecuador es de 3 a 1 a favor del primero (basado en las importaciones realizadas en el 2006). Santander se ha enfocado en exportar calzado principalmente a Ecuador. A Venezuela el volumen de exportación del sector ha sido más bajo. 
Un estudio especial requiere analizar la aparente contradicción dada entre el registro en bases de datos sobre las exportaciones de Santander a Venezuela (en comparación con Ecuador) y los datos que proporciona la información primaria. Las cifras estadísticas muestran mayores exportaciones a Ecuador (en unidad monetaria). Los datos arrojados por la encuesta dejan ver mayores exportaciones de los empresarios a Venezuela que a Ecuador (en pares exportados).

\section{Referencias}

Aranda, Y.; Montoya V. (2006). Principales enfoques conceptuales explicativos del proceso de internacionalización de empresas. Agronomía Colombiana 24(1): 170-181

Bekerman, M.; Sirlin, P. (2000). Impactos estáticos y dinámicos del Mercosur. El caso del sector calzado. Revista de la Cepal. 72. Diciembre 2000. 185 - 2002.

Conindustria (2005). Coyuntura semanal. Centro de estudios económicos y legales (Venezuela). Coninnceel. 27

Czinkota M.; Ronkainen I. (2007). Negocios Internacionales. México. México Cengage Learning Editores.

López, R. (2005). Logística comercial. Madrid. España. Thomson editores

Olivares A.; Suárez S. (2006). El proceso exportador de las empresas manufactureras españolas. Revista Europea de Dirección y Economía de la empresa. 16, 89 - 98

Porter, M. (1991). La ventaja competitiva de las naciones. Buenos Aires. Argentina. Javier Vergara Editor

Soret, I. (2004). Logística comercial y empresarial. Madrid. España. Esic. 